\title{
Critical incident reporting: learning from errors to improve patient safety
}

Irene Harth PN; FIP, Johannes Gutenberg Universität Mainz, Klinik für Kinder-und Jugendmedizin, PICU 109 AE 2, 55101 Mainz, Germany

E-mail: iharth@onlinehome.de

Key Words: critical incident reporting * medical error $\quad$ open safety culture $\quad$ risk management

\section{SUMMARY}

- Over the last decade patient safety has become an important focus of interest in all health care areas.

- Reducing errors in order to improve quality and lower costs in hospitals has become one of the main efforts of risk management.

- System analysis identifies failures within the whole system to be responsible for critical events occurring in hospitals.

- Multiple factors on different system levels contribute to the complexity of medical errors; the human risk factor is only one facet of the problem.

- Critical incident reporting, as an element of risk management, allows the identification of contributing factors to risk situations, reported by personnel directly involved in the process. Subsequent root cause analysis will lead to the proposal of changes to improve performance and to avoid future critical events.

- $\quad$ Although there are obvious benefits to incident reporting, most hospital reporting systems fail to detect the majority of critical events and some long-lasting misconceptions and scepticisms exist.

- This paper outlines the basic conditions for a successful incident reporting system and discusses why these systems are still not accepted by some health care professionals.

\section{INTRODUCTION}

"If you feel safer in a hospital than on an airplane - think again! Paradoxically people are more frightened of air travel than they are in healthcare," said Sir Liam Donaldson, Chair of World Health Organization World Alliance for Patient Safety during a conference on patient safety in London in November 2005. He explained that the risk of being killed in an air crash is one in ten million compared to the risk of dying during a stay in a hospital of the western world, which is one in 300 (Medscape, 2005).

Consistent with this, the UK Department of Health (2000) indicated that adverse events occur in about $10 \%$ of all hospital admissions per year. Furthermore, research results published by New Zealand and Canadian working groups confirm this high rate of adverse events (New Zealand Ministry of Health, 2001; Baker et al., 2004). Thus, it is not surprising that $35 \%$ of physicians and $42 \%$ of patients or relatives report that they have experienced an error in their own care or in that of a family member when they were asked about their experiences with medical errors (Blendon et al., 2002). Moreover, $18 \%$ of physicians and $24 \%$ of the public have reported an error that has had serious health consequences including death, long-term disability and severe pain (Blendon et al., 2002).

\section{Economic impact of critical incidents}

Adverse events are cost-intensive; not only from a financial point of view. Errors also incur costs which often cannot be measured directly but manifest as decreased patient satisfaction and reduced trust in the health care system.

In the annual health report 2000 for Germany the Robert Koch Institut (2001) in Berlin estimated that an average of 550 DM additional costs per hospital bed were due to costs that insurance companies had to pay for compensation due to medical errors in 1999. More recently, these figures have risen by up to $400-1500$ euro per bed, with the consequence that some hospitals cannot afford to pay their liability insurances anymore (Ghanaat \& Goslich, 2003).

It is also important to acknowledge the influence of a negative press or word of mouth when medical errors occur. For example, in an orthopaedic hospital in the south of Germany that there was a decrease of $9 \%$ in patient admissions following a report about a medical mishap in the local press (Bernsmann et al., 2002). In addition, the German news magazine, The Focus, which assesses hospitals concerning their performance according to certain criteria, and publishes an annual hospital ranking system, has the power to influence the number of patient admissions (Bernsmann et al., 2002). In today's economic climate adverse publicity has the power to accelerate the fusion of competitive hospitals or can precipitate the closure of whole departments in hospitals or even the hospital itself. Thus, reducing medical management errors has become a central issue in the drive to improve quality and lower costs in health care.

\section{System and safety culture}

In the Institute of Medicine (2000) report To Err is Human - Building a Safer Health System it was stated that medical errors should be viewed as failures of institutional systems rather than failures of individuals. They should be seen in a larger context in which several factors came together at the same time that then led to an error occurring. Also, it is important to recognise that the human element is only one aspect of these complex systems. Therefore, system analysis is one of the main concepts applied in managing in patient safety. This approach focuses primarily on system organisation and processes; it considers the conditions under which individuals work.

The popular Swiss Cheese Model (Figure 1) developed by James Reason characterises the latent failures present on different levels in health care systems. Defences and barriers at organisational, managerial, technological and individual levels are compared with slices of Swiss cheese. The holes in the slices of the cheese represent the problems on different levels and each single factor might contribute to the failure of the overall system. This model enables the division of a critical event into single steps in order to identify all contributing factors at each level of the system (Reason, 2000) 


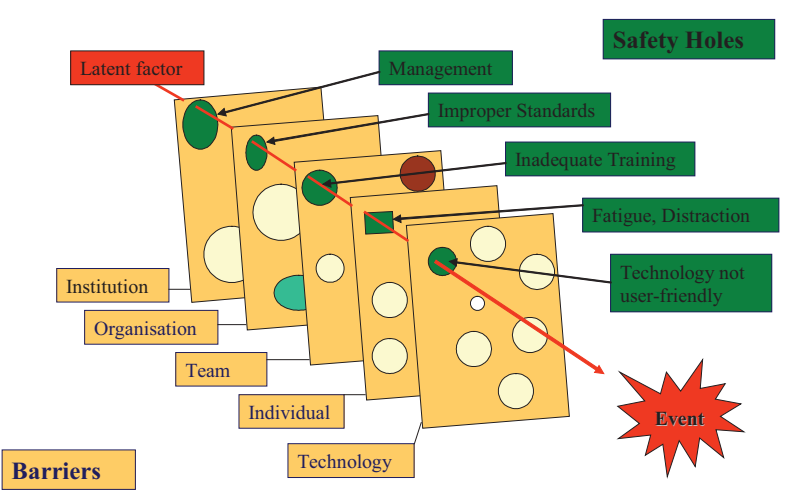

Figure 1. The Swiss Cheese Model (adapted)

\section{CRITICAL INCIDENT REPORTING}

Critical incident reporting is an element of risk management which involves:

- the identification of preventable events, reported by personnel directly involved in the process,

root cause analysis,

- the proposal of changes to avoid similar events in the future.

Incident reporting may targeted on adverse events and critical incidents but the real focus of interest is set on 'near misses'. Near misses are defined as any event that could have had adverse consequences but did not. These events are less likely to provoke guilt or other psychological barriers to reporting.

With critical incident reporting, all healthcare providers are requested to report anonymously about critical situations, which they experience in their daily practice. The reported data should integrate only elements that identify:

\footnotetext{
- the type and experience of personnel involved in the incident,

- a description of the incident,

- patient details and complaints.
}

The person who reports the incident is also invited to submit suggestions on the factors that possibly contributed to the incident, to describe possible corrective actions which have been undertaken, and offer ideas about how to prevent similar events in the future.

Quality managers evaluate the collected data. This process requires a thorough understanding of the details of the task, the context, the environment and its constraints. Ideally, experts with an interdisciplinary professional background should look at these reports to identify basic or causal factors. In this context, it is not helpful to construct taxonomies of incidents as this only permits quantification. This may be one of the reasons why critical incident reporting has not become standard procedure in many hospitals; many systems focus more on gathering statistical data than on system analysis.

With system analysis several basic questions are posed:

\section{- How did the incident happen?}

- Which factors contributed to this incident on which level?

- Which safety barriers were passed?

- Which strategy to intervene was used?

The safety 'holes' mentioned above have to be identified, described and closed with specific actions. Together with the team involved the quality managers should develop proposals for system and process changes in order to improve performance and to reduce the potential for future events. Furthermore, neutral descriptions of the past events should be available for every team member. It may be helpful to also provide a short summary of each critical incident so that it can be viewed via the hospital's intranet for educational or informational reasons. It is important that when using critical incidents to provide feedback to clinical teams and for educational reasons that a 'no blame' approach is taken. This requires a neutral non-judgemental description of the incident and the actions that were implemented to improve performance. Thus, each team member gets the chance to learn from mistakes others have already made. Rapid and meaningful feedback from quality managers to all interested or involved parties is the most important factor in determining the quality of incident reports and the success of a reporting system. The responding system is just as important as the reporting system (World Alliance for Patient Safety, 2005).

\section{Benefits and pitfalls of critical incident reporting}

Our experience of managing critical incident reporting systems has shown that the smaller the radius of the system, the better it works. For example, a regional system works better than a national or international system, and a hospital system works better than a regional system. This is because a more immediate and individual response can be made (Institute for Safe Medication Practice, 2003). However, a central organisation for incident reporting does have the advantage that it would be more likely to discover trends. Also, when systematically evaluating aggregated reported data there may be greater potential to influence change on a political level (Barach \& Small, 2000).

Several benefits of using incident reporting systems have been identified:

- $\quad$ system analysis by experts may enhance process improvement through the implementation of safety initiatives,

- it contributes to better risk management planning as safety holes are detected,

- hospital or unit managers, when using the data, are able to identify areas where additional training programmes are needed,

- a growing sensitivity among the health care professionals to detect critical situations in a preliminary stage has been noted, which may lead to the enhancement of proactive interventions.

\section{Why is critical incident reporting under-used?}

Most incident reporting systems in hospitals are facing the problem of under-reporting (Barach \& Small, 2000). The Institute for Safe Medication Practice (2003) reported the results of a survey about the intentions of health care practitioners to report incidents, such as medication safety issues, to a critical incident reporting programme. They found that the strongest motivational factors among nurses were that the information was used to enhance safety and to prevent needless tragedies. However, the survey results also indicated that some healthcare providers in key positions, such as academics and staff educators, senior leaders and managers were not well-informed about reporting programmes. This was seen as an issue of concern because such people are often in the best position to influence policy and to teach the coming generations of healthcare professionals about the value of error reporting systems (Institute for Safe Medication Practice, 2003). Results from a more recent survey (Taylor et al., 2004) about the use of incident reports by physicians and nurses to document medical errors in paediatric patients showed that a lack of certainty about what is defined as an error and concerns about implicating others were key reasons for under-reporting.

Further disincentives to reporting include the perception that it involves extra work, fear of reprisals, loss of reputation and, in the worst case, the loss of job. Another reason for non-reporting can be the perceived lack of effectiveness of the reporting system (Barach \& Small, 2000). These factors vary considerably depending upon the organisational culture of a system, with anonymous or non-punitive systems being more likely to increase the rate of reporting.

A principal condition for a successful incident reporting system is that 
there is a consensus on the managerial level upon the need for better information on safety issues. Also critical is the implementation of an independent group to collect and analyse reporting data. This group should be an organisationally-respected body and must have adequate time to work on the system. Interventions that help to increase the likelihood of critical incident reporting include ongoing education about the nature of errors which should be reported. As mentioned above, rapid and meaningful feedback on a regular basis to all involved parties about the reported error and the lessons learned is the most important factor in determining the quality of incident reports (Taylor et al., 2004).

\section{CONCLUSIONS}

Critical incident reporting, properly interpreted, provides knowledge. Using this knowledge to improve the performance of a system in order to improve patient safety is the fundamental advantage of critical incident reporting. In the overall context, critical incident reporting may save lives, property and resources and reduce injuries. Therefore, it is important that efforts are made to promote and improve reporting.

This may include initiatives to educate all healthcare providers about the types of incidents that should be reported. The reporting system must be easily accessible and regular feedback on the reported errors and the subsequent system changes are basic requirements for the success of the system.

Critical incident reporting works well when it is an easy to do, safe and effective procedure for all healthcare professionals. Easy means that the system requires no undue use of time and extra work; this could be achieved using an electronic system. Safe means that the system is trusted and that healthcare staff have no fear of reprisals. Effective means that there is an obvious benefit for the people involved.

However, critical incident reporting is just one element of a good hospital clinical governance programme, which should be embedded within an organisational culture that continuously strengthens patient safety.

\section{REFERENCES}

Baker GR, Norton PG, Flintoft V, Blais R, Brown A, Cox J, Etchells E, Ghali WA, Hebert P, Majumdar SR, O'Beirne M, Palacios-Derflingher L, Reid RJ, Sheps S, Tamblyn R (2004). The Canadian Adverse Events Study: the incidence of adverse events among hospital patients in Canada. Canadian Medical Association Journal170 (11), 1678-1686.

Barach P, Small S (2000). Reporting and preventing medical mishaps: lessons from non-medical near miss reporting systems. BMJ 320 : 759-763.
Bernsmann K, Neumann M, Schleberger R (2002). Risikomanagement in der Krankenhauspraxis [Risk Management in Hospital Practice]. Stuttgart, Verlag W. Kohlhammer.

Blendon RJ, DesRoches CM, Brodie M, Benson JM, Rosen AB, Schneider E, Altman DE, Zapert K, Herrmann MJ, Steffenson AE (2002). Views of practicing physicians and the public on medical errors. New England Journal of Medicine 347 (24), 1933-1940.

Department of Health (2000). An Organisation With Memory: Report of an expert group learning from in the NHS chaired by the Chief Medical Officer (online). Available at: http://www.dh.gov.uk/en/ Publicationsandstatistics/Lettersandcirculars/Dearcolleagueletters/ DH_4005264

Ghanaat H, Goslich L (2003). Risikomanagement - Aus Fehlern lernen [Risk management - learn from errors]. Klinik Management Aktuell 87, 26-34.

Institute for Safe Medication Practice (2003). ISMP Survey on Internal and External Medication Error Reporting (online). Available at: http:// www.ismp.org/Survey/NewsLetter/Survey11122002Results.htm

Institute of Medicine (2000). To err is human: building a safer health system (online). Available at: http://www.nap.edu/books/0309068371/ html/

Medscape (2005). Hospitals Told to Learn Safety Rules From Airlines (online). Available at: http://www.medscape.com/viewarticle/517999

New Zealand Ministry of Health (2001). Adverse Events in New Zealand Public Hospitals: Principal Findings from a National Survey (online). Available at: http://www.moh.govt.nz/publications/adverseevents

Reason J (2000). Human error: models and management. BMJ 320, 768-770.

Robert Koch Institut (2001). Medizinische Behandlungsfehler: Gesundheitsberichterstattung des Bundes [Medical Treatment Errors: Health Reporting of the Federation]. Robert Koch Institut, Statistisches Bundesamt [Federal Office of Statistics] 04/01, 7.

Taylor JA, Brownstein D, Christakis DA, Blackburn S, Strandjord P, Klein EJ, Shafii J (2004). Use of incident reports by physicians and nurses to document medical errors in pediatric patients. Pediatrics 114 (3), 729-735.

World Alliance for Patient Safety (2005). Forward Programme 2005: Reporting and Learning to Improve Patient Safety (online). Available at: http://www.who.int/patientsafety 